\title{
ULTRASONIC VERIFICATION OF MICROSTRUCTURAL CHANGES DUE TO HEAT TREATMENT
}

\author{
Edward R. Generazio \\ National Aeronautics and Space Administration \\ Lewis Research Center \\ Cleveland, Ohto 44135
}

\begin{abstract}
Ultrasonic attenuation was measured for polycrystalline samples of nickel and copper with varlous grain-size distributions produced by heat treatment. Attenuation as a function of frequency was determined for a sample having a known mean grain diameter. Once this function was determined, it could be scaled to determine the mean grain size of other samples of the same material with different mean grain diameters. These results were obtained by using broadband pulse-echo ultrasound in the 25- to $100-\mathrm{MHz}$ frequency range. The results suggest an ultrasonic, nondestructive approach for verifying heat treatment of metals.
\end{abstract}

\section{INTRODUCTION}

An extensive effort has been made to relate the observed uitrasonic attenuation to the mean grain diameter in polycrystalline solids (refs. 1 to 7 ). Although the experimental and theoretical results agree for some polycrystalline materials, many materials are so complex that they are not adequately described by existing theory (refs. 8 to 13). Because structural materials generally exhibit complex microstructures, verification of their microstructures is of high interest. As a practical matter, determining the mean grain size of both the simple and complex systems by nondestructive ultrasonic attenuation is preferable to the usual time-consuming, expensive, and inherently destructive metallographic methods.

Most polycrystalline solids are a collection of densely packed, inhomogeneous, and morphologically complicated scatters. Previous investigators have usualiy assumed that a particular mode of scatter attenuation (Rayleigh, stochastic, etc.) is dominant when comparing experimental and theoretical results. However, in many complex systems both Rayleigh and stochastic scattering may occur for a given range of frequencies (or wavelengths).

In general Rayleigh, stochastic, and diffusive scattering may occur simultaneously. The relative contribution of each scattering mechanism to the total observed attenuation is unknown for a complex microstructure. In this study no attempt was made to attribute attenuation to a particular scattering mode. We merely observed that a significant factor in comparing changes in microstructure is a ratio containing wavelength and mean grain diameter. It will be shown that any arbitrary wavelength can be used to establish a mean-grain-sizerelated scale factor between two polycrystalline microstructures. 
Expressions for uitrasonic attenuation coefficient $\alpha$ as a function of frequency $f$ have been developed for three regimes: the Rayleigh scattering regime, where wavelength $\lambda$ greatly exceeds mean grain size $\bar{D}(\lambda \gg \bar{D})$

$$
\alpha_{R}=C_{R} \bar{D}^{3} f^{4}, \mathrm{~Np} / \mathrm{Cm}
$$

the stochastic scattering regime, where $\lambda \simeq \overline{\mathrm{D}}$,

$$
\alpha_{s}=C_{s} \bar{D} f^{2}, \mathrm{~Np} / \mathrm{cm}
$$

and the diffusion scattering regime, where $\lambda \ll \bar{D}$,

$$
\alpha_{d}=\frac{C_{d}}{D}, \mathrm{~Np} / \mathrm{Cm}
$$

Here, $C_{R}, C_{S}$, and $C_{d}$ are constants that depend on material parameters such as elastic constants and longitudinal and shear velocities. Typical dimensions for a are nepers per centimeter. Equations (1) to (3) are generally valid only for homogeneous, regular, and equiaxed systems (ref. 10).

Note that each of the expressions (eqs. (1) to (3)) contains a coefficient that depends on $\bar{D}$, the mean grain size. Assume that the constants $C_{R}, C_{S}$, and $C_{d}$ are unaffected by changes in $\overline{\bar{D}}$. Also assume that the shape of the grain size distribution function and grain morphology are unaltered by changes in $\bar{D}$. Given these assumptions, the microstructure would simply change by a scale factor corresponding to the increase (or decrease) in $\bar{D}$. Substituting, $V / \lambda$, where $V$ denotes velocity, for $f$ and multiplying equations (1) to (3) by wavelength $\lambda$ yields a new set of equations in which $\bar{D} / \lambda$ is the key variable. Thus, given that velocity is not a function of frequency, we have for Rayleigh scattering

$$
\alpha_{\lambda}=C_{R}^{\prime}\left(\frac{\overline{0}}{\lambda}\right)^{3}, \text { Np/wave length }
$$

for stochastic scattering

$$
a_{\lambda}=C_{s}^{\prime}\left(\frac{\bar{D}}{\lambda}\right), N p / \text { wavelength }
$$

for diffusion scattering,

$$
a_{\lambda}=C_{d}^{\prime}\left(\frac{\lambda}{\delta}\right) \text {, Np/wave length }
$$

where $C_{R}^{\prime}, C_{s}^{\prime}$, and $C_{d}^{\prime}$ are constants. The attenuation coefficient is now expressed in nepers per wavelength for each regime. 
Evaluating equation (5) at some arbitrary wavelength $\lambda_{0}$ for a system $S_{0}$ having a mean grain diameter $\bar{D}_{0}$ (fig. $\left.1(a)\right)$, we obtain the attenuation coeffictent

$$
a_{\lambda}=\left.c_{s}^{\prime}\left(\frac{D}{\lambda}\right)\right|_{\delta_{0}, \lambda_{0}, c_{s}^{\prime}}=\alpha_{0} \text {, Np/wavelength }
$$

If the mean grain size and the wavelength are doubled (fig. $1(b)$ ), we obtain from equation (5),

$$
\alpha_{\lambda}=\left.c_{s}^{\prime}\left(\frac{\bar{D}}{\lambda}\right)\right|_{2 \bar{D}_{0}, 2 \lambda_{0}, c_{s}^{\prime}}=\alpha_{0} \text {, Np/wavelength }
$$

or the same numerical value for the attenuation coefficient as obtained in the previous case (simllar results can be obtained by using eq. (4) or (6)). This is shown graphically in figure 2(a). By varying the arbitrary wavelength $\lambda_{0}$, we can construct the attenuation-versus-wavelength functions $S_{0}$ and $S$ (fig. $2(b)$ ). Because the mean grain size of system $S$ is also arbitrary, we can construct the attenuation-versus-wavelength function for the $\mathrm{N}^{\text {th }}$ system $S_{n}$ having the mean grain size $\bar{D}_{n}$.

A scale factor can be defined as

$$
\sigma_{n} \equiv \frac{\lambda_{n}}{\lambda_{0}}=\frac{\delta_{n}}{\delta_{0}}=N^{\text {th }} \text { scale factor }
$$

where $\bar{D}_{n} / \bar{D}_{0}$ is the ratio of the mean grain size of the $N^{\text {th }}$ system to the mean grain size of the initial system. The scale factor is also represented as the ratio of wavelengths for which constant attenuation an is observed between two systems (1.e.. the ratio of wavelength $\lambda_{n}$ of the $N$ th system to the wavelength $\lambda_{0}$ of the initial system at constant attenuation).

The curve $s_{0}$ in figure $2(b)$ is the generating function from which the other curves can be obtained. To construct the generating function, a fourthdegree polynomial in frequency was fitted to the attenuation data by using the method developed by Marquardt (refs. 14 and 15). Here the attenuation is expressed as

$$
\alpha_{0}(f)=\sum_{m=1}^{4} A_{m} f^{m}, N p / c m
$$

where $A_{m}$ are constants. The terms in the expansion shown in equation (10) are not directly related to equations (1) to (3). Any other analytical expression that fits the attenuation data would be equally valid. The preceding curve-fitting function is made a function of wavelength by combining equation 10 with 


$$
V=f \lambda
$$

where $V$ is the velocity of sound, to obtain

$$
\alpha_{0}(\lambda)=\sum_{m=1}^{4} A_{m}^{\prime}\left(\frac{1}{\lambda}\right)^{m}, \mathrm{~Np} / \mathrm{cm}
$$

where $A_{m}^{\prime}$ are constants. By multiplying equation (12) by the variable wavelength, we obtain the generating function

$$
\alpha_{\lambda}=\sum_{m=1}^{4} A_{m}^{\prime}\left(\frac{1}{\lambda}\right)^{m-1} \text {, Np/wavelength }
$$

The theoretical attenuation for other systems (of larger grain size) is obtained from their respective generating functions by scaling the wavelength as indicated in figure 2. The scale factor $\sigma$ is varied until the generating function for the initial system with the scaled wavelengths reconstructs the attenuation for the $N$ th system. If the mean grain size of the initial system is known, the mean grain size $\bar{D}_{n}$ for the $N^{t h}$ system is given by equation (9).

\section{EXPERIMENT}

\section{Material Samples}

Two materials, nickel 200 and copper, were used in this investigation. The nickel 200 was 99.5 percent pure and the copper was 99.99 percent pure. Both were obtained as commerclally avallable rods. The nickel 200 was then cold rolled 50 percent and annealed in air at $690 \pm 9{ }^{\circ} \mathrm{C}$ and cold rolled again 50 percent. The copper sample was cold rolled 60 percent and annealed at $329^{\circ} \mathrm{C}$ in argon for $5 \mathrm{~min}$. Three nickel samples, cut from the rolled stock, had typical dimensions of 1 by 1 by $0.2540 \mathrm{~cm}$. Five copper samples, cut from the rolled stock, had typical dimensions of 1.2 by 0.8 by $0.18 \mathrm{~cm}$. Each sample was polished to a mirror finish with a final polish of 1.0-um-diameter diamond slurry.

\section{Microstructure Modification}

The mean grain size was increased in both the nickel and copper samples by annealing (refs. 16 and 17). Three nickel samples were annealed in atr at $800{ }^{\circ} \mathrm{C}$ for 1,2 , and $5 \mathrm{~min}$, respectively. Four of the five copper samples were annealed in argon for $5 \mathrm{~min}$ at $466,510,700$, and $800^{\circ} \mathrm{C}$, respectively. 


\section{Attenuation Measurement}

Before modifying the initial grain size, we made attenuation measurements on each sample. The methodology for making precision attenuation measurements is described in reference 18. These initial attenuation measurements were made to verify that all of the nickel and copper samples were nearly acoustically identical to the other samples of the same material. At $50 \mathrm{MHz}$ there was 4 and 6 percent variation in the attenuation measurement among nickel and copper samples, respectively. These variations included the effect of the imprecision in attenuation measurements that include the frequency-dependent reflection coefficient in the calculation (ref. 18).

After the mean grain size had been modified, attenuation measurements were again made on each sample. Since the attenuation coefficient (at fixed frequency) generally increases with mean grain size, samples exhibiting extremely high attenuation were ground thinner so that the uncertainty in the attenuation measurement was minimized (ref. 18). The results were reproducible with typical variations in measurement simflar to those for the initial attenuation measurement.

\section{RESULTS \\ Experimental Generating Functions}

In general, for a fixed frequency, the attenuation coefficient increased with annealing time (fig. 3 ). The solid curve is the best-fit curve (i.e., generating function) obtained by using equation (10), for the data for the nickel sample annealed for $1 \mathrm{~min}$. The data shown in figure 3 reappear in figure 4 as a function of wavelength. The solid curves for the samples annealed for 2 and $5 \mathrm{~min}$ were determined from the generating function by scaling the wavelength, as discussed earlier. The observed and theoretical results are shown in figure 5 as a function of frequency.

For fixed frequency the attenuation coefficient increased with annealing temperature (fig. 6) for the copper samples. The solid curve for the copper sample annealed at $329^{\circ} \mathrm{C}$ is the generating function for these samples. The data shown in figure 6 reappear in figure 7 as a function of wavelength. The solid curves were determined from the generating function by scaling the wavelength. The observed and theoretical results are shown in figure 8 as a function of frequency.

The scale factors for the data shown in figures 4 to 8 were determined by scaling the generating function to fit the frequency regime for which the most accurate data were obtained. This regime, although well defined (ref. 18), was different for each sample and depended on the sample thickness, the attenuation, and the frequency-dependent reflection coefficient.

Deviations existed between the experimental and theoretical results (figs. 4 to 8 ) at both the low and high frequencies ( $1 . e .$, long and short wavelengths). These deviations were due to the greater uncertainty in the measurement of attenuation at these extreme frequencies (ref. 18). 
Several methods are avallable for determining the mean grain size (refs. 12 and 19). For regular systems the standard line-intercept method appears to be adequate. However, this method is not applicable to systems with structuraliy complicated morphologies. More advanced theories that are based on spherical grains also are inherently not applicable to real, complicated systems. An alternative technique, the comparison method (ref. 19), is free of the theoretical bias imposed by the above-mentioned theories.

The simple comparison method allows for the grain structure to be visually compared with an ASTM standard chart. This technique is not directly applicable to complicated systems (i.e., the standard ASTM charts are for regular structures). We can modify the comparison method so that we may compare systems with dimensionally arbitrary scales but of similar complexity. This is done by taking photomicrographs of similar systems of different scales and subsequentiy magnifying the photomicrograph for the smaller system by the appropriate scale factor between the two systems. The magnified photomicrograph and the photomicrograph for the system of larger scale should appear similar. This is shown graphically in figures 9 and 10 for the nickel and copper samples, respectively. (Uitrasonic attenuation was measured along the vertical axis.)

From figure 4, the scale factor between the photomicrographs for the nickel systems annealed for 2 and $5 \mathrm{~min}$ (figs. 9(a) and (b)) is 1.85 . Figure $9(a)$ is shown magnified 1.85 times in figure $9(\mathrm{c})$, which is dimensionally and morphologically similar to figure $9(b)$. Similar results are shown in figure 10 for the copper samples annealed at 510 and $800^{\circ} \mathrm{C}$. Comparisons with the other systems show strikingly similar results.

\section{DISCUSSION}

The success of scaling a generating function over a wide frequency range to other systems of similar complexity but of different scale was quite remarkable in that only one free parameter was needed. This parameter is directly related to the relative scale between systems. Ideally, one would like to use (scale) a theoretical expression for uitrasonic attenuation that includes multiple grain-boundary scattering for morphologically complicated systems. However, such an expression is currentiy unavallable since the theory of grainboundary scattering for complicated systems remains underdeveloped (ref. 10).

The lack of a comprehensive theory can be bypassed by measuring the attenuation for an initial system as a function of frequency. The effects of grain distribution, morphology, orientation, and grain interaction are implicit. This experimentally measured attenuation also includes attenuation due to dislocation damping (refs. 6 and 20), thermoelastic effects (ref. 20), etc. These nonbarrier attenuation mechanisms will not scale as the grain-boundary scattering terms (eqs. (4) to (6)) do. We have assumed in the preceding analysis that these unscalable attenuation mechanisms are negligible relative to the observed attenuation. This, however, will not be true for materials that are in a mechanically worked state, where the dislocation densities are expected to be large (ref. 16). 
A major requirement for the scaling method to be successful is that the systems of interest must have similar morphology. This is a somewhat subjective judgment not quantitatively described. The lack of a comprehensive theory for determining the mean grain size for a real, complicated system has left us with no alternative, other than the comparison method, for making quantitative comparisons between theory and experiment. Although magnifying the photomicrographs (figs. 9 and 10 ) served to make these comparisons, it is not the best method for comparing grain structures. A more precise method would be to use a continuous ly variable magnification metallograph.

The theorles for both ultrasonic boundary scattering and grain size distribution are insufficientiy developed to provide quantitative results for comparing experiment and theory. This problem is temporarily resolved by using the observed attenuation and the observed microstructure to show this agreement. The full comprehensive theory for the ultrasonic attenuation that includes grain-boundary scattering for morphologically complicated systems and allows for interacting grains is expected to scale as the experimental results indicate here.

The simple concept of mean grain size is likely to be inappropriate for both acoustic and microstructure theory. A more complete description of the microstructure morphology would include the distribution of grain size (ref. 12) and grain boundary topology. This more complete description should be included in determining of the microstructure morphology and ultrasonic attenuation theory. Nevertheless, even in the form described in this paper the approach for determining microstructure scale is likely to be useful in verifying the heat treatment of most polycrystalline materials.

\section{CONCLUSIONS}

It has been shown that the ratio between wavelength and mean grain diameter is a significant factor in comparing changes in microstructure. This ratio can be used to determine a scale factor between two polycrystalline materials with similar morphologies but different mean grain diameters. These results were achieved by determining the attenuation as a function of frequency without reference to any particular conventionally accepted scattering mode. By this approach it was unnecessary to associate a particular wavelength with mean grain diameter. This allowed a generalized formulation for scaling meangrain-diameter variations among samples of a polycrystalline material that had undergone a range of heat treatments.

\section{REFERENCES}

1. Evans, A.G., et al.: Fallure Prediction in Structural Ceramics, Mater. Eval., vol. 35, no. 4, Apr. 1977, pp. 85-96.

2. Kesler, H.A.; and Shraifeld L.I.: Dispersion of Ultrasonic Waves in Polycrystalline Metals with Statistically Distributed Grain Size, Sov. J. Nondestr. Test (Engl. Trans1.), vol. 11, no. 1, Nov. 1975, pp. 76-80. 
3. Papadakis, Emmanuel P.: Ultrasonic Attenuation Caused by Scattering Polycrystalline Metals, J. Acoust. Soc. Am., vol. 37, no. 4, Apr. 1965, pp. $711-717$.

4. Serabian, S.; and Williams R.S: Experimental Determination of Ultrasonic Attenuation Characteristics Using the Roney Generalized Theory, Mater. Eval., vol. 36, no. 8; July 1978, pp. 55-62.

5. Papadakis, E.P.: Some Practical Aspects of Research with U1trasonic Waves, Int. J. Nondestr. Test., vol. 1, 1970, pp. 383-399.

6. Smith, R.L., et a1.: Ultrasonic Attenuation, Microstructure, and Ductile to Brittle Transition Temperature in Fe-C Alloys, Mater. Eva1., vol. 41, no. 2, Feb. 1983, pp. 219-222.

7. Klinman, R., et al.: Ultrasonic Prediction of Grain Size, Strength, and Toughness in Plain Carbon Stee 1, Mater. Eval., vol. 38, no. 10, Oct. 1980, pp. 26-32.

8. Bhatia, A.B.: Scattering of High-Frequency Sound Waves in Polycrystalline Materials, J. Acoust. Soc. Am., vol. 31, no. 1, Jan. 1959, pp. 16-23.

9. Bhatia, A.B.; and Moore, R.A.: Scattering of High Frequency Sound Waves in Polycrystalline Materials II, J. Acoust. Soc. Am., vol. 31, no. 8, Aug. 1959, pp. 1140-1141.

10. Papadakis, Emmanuel P.: Ultrasonic Attenuation Caused by Rayleigh Scattering by Graphite Modules in Modular Cast Iron, J. Acoust. Soc. Am., vo1. 70, no. 3, Sept. 1981, pp. 782-787.

11. Roth, W.: Scattering of Ultrasonic Radiation in Polycrystalline Metals, J. App 1. Phys., vol. 19, no. 10, Oct. 1948, pp. 901-910.

12. Papadakis, E.P.: From Micrograph to Grain-Size Distribution with U1trasonic Applications, J. Appl. Phys., vol. 35, no. 5, May 1964, pp. 1586-1594.

13. Smith, R.L.; Reynolds, W.N.; and Wadley, H.N.G.: Ultrasonic Attenuation and Microstructure in Low Carbon Stee1s, Met. Sc1., vol. 15, no. 11-12, Nov.-Dec. 1981, pp. 554-558.

14. Marquardt, D.W.: An Algorithm for Least-Squares Estimation of Nonlinear Parameters, J. Soc. Ind. App 1. Math., vol. 11, no. 2, 1963, pp. 431-441.

15. Bevington, R.P.: Data Reduction and Error Analysis for the Physical Sclences, Chapter Four, McGraw-Hi11, New York, 1969.

16. Dieter, G.E.: Mechanical Metallurgy, Chapter 5, McGraw-H 111 , New York, 1961 .

17. Metals Handbook, eighth Ed., vol. 7, American Society for Metals, Metals Park, Ohio, 1972. 


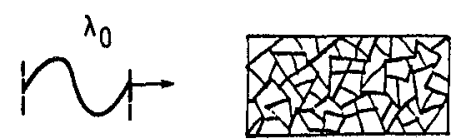

(a) Initial system $S_{0}$ with mean grain diameter $\bar{D}_{0}$.<smiles>[Te][Te]</smiles>
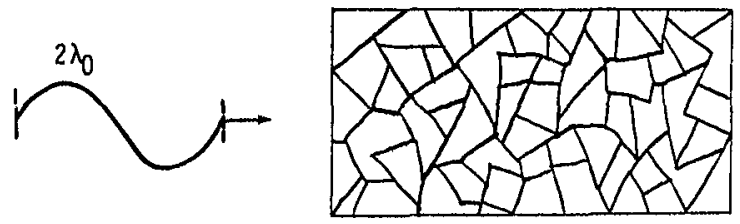

(b) Scaled system $\mathrm{S}$ with mean grain diameter $2 \bar{D}_{0}$.

Figure 1. - Initial and scaled systems.

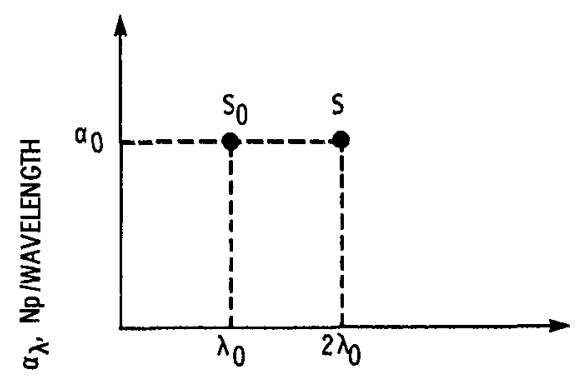

(a) Systems $S_{0}$ and $S$ at fixed wavelengths.

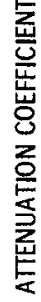

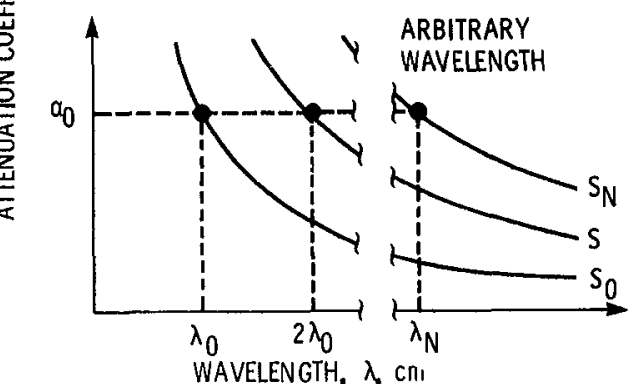

(b) Systems $S_{0}, S$, and $S_{N}$. Curve labeled $S_{0}$ is generating function.

Figure 2. - Attenuation coefficient as function of wavelength.

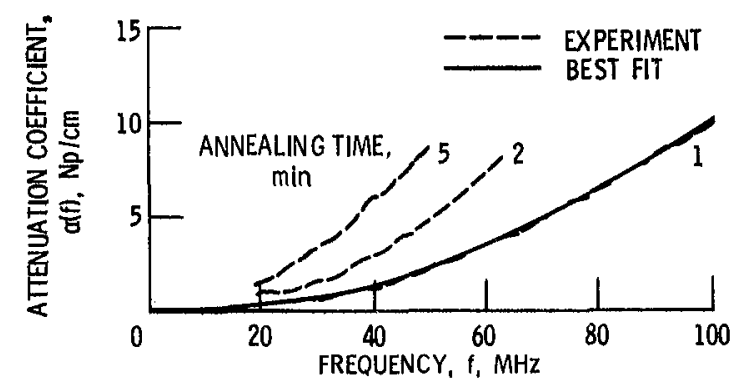

Figure 3. - Atten uation coefficient as function of frequency for nickel samples. Annealing temperature, $800^{\circ} \mathrm{C}$. Solid curve is generating function.

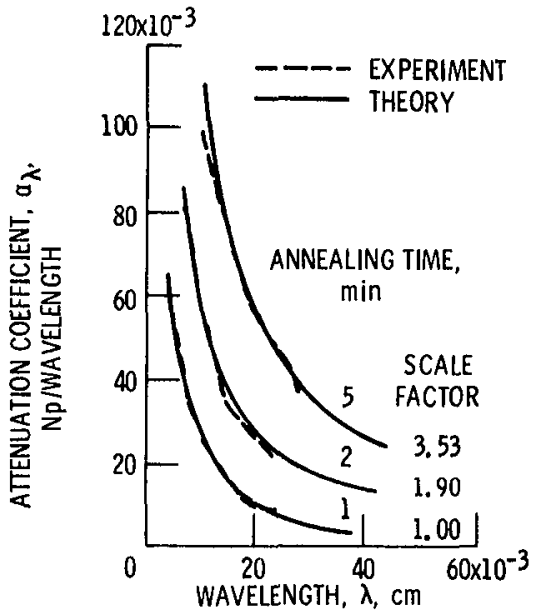

Figure 4. - Attenuation coefficient as function of wavelength for nickel samples. Annealing temperature, $800^{\circ} \mathrm{C}$. 


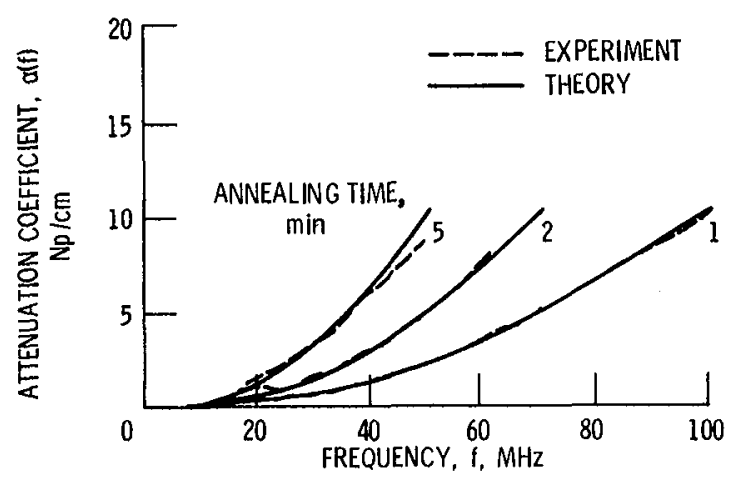

Figure 5. - Comparison between observed and theoretical attenuation coefficients as function of frequency for nickel samples. Annealing temperature, $800^{\circ} \mathrm{C}$.

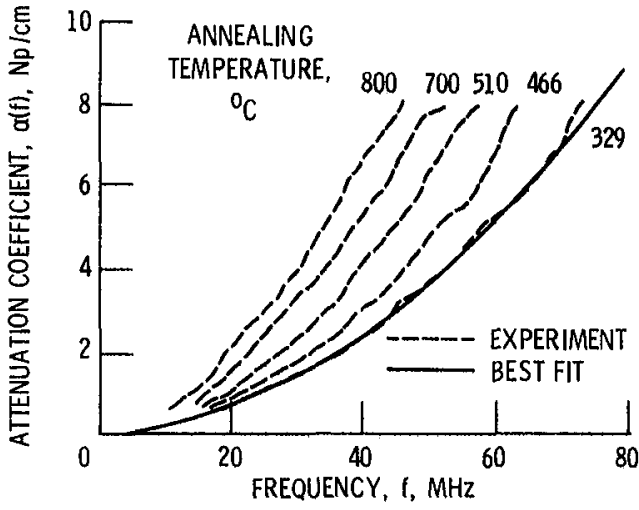

Figure 6. - Atten uation coefficients as function of frequency for copper samples. Annealing time, $5 \mathrm{~min}$. Solid curve is generating function.

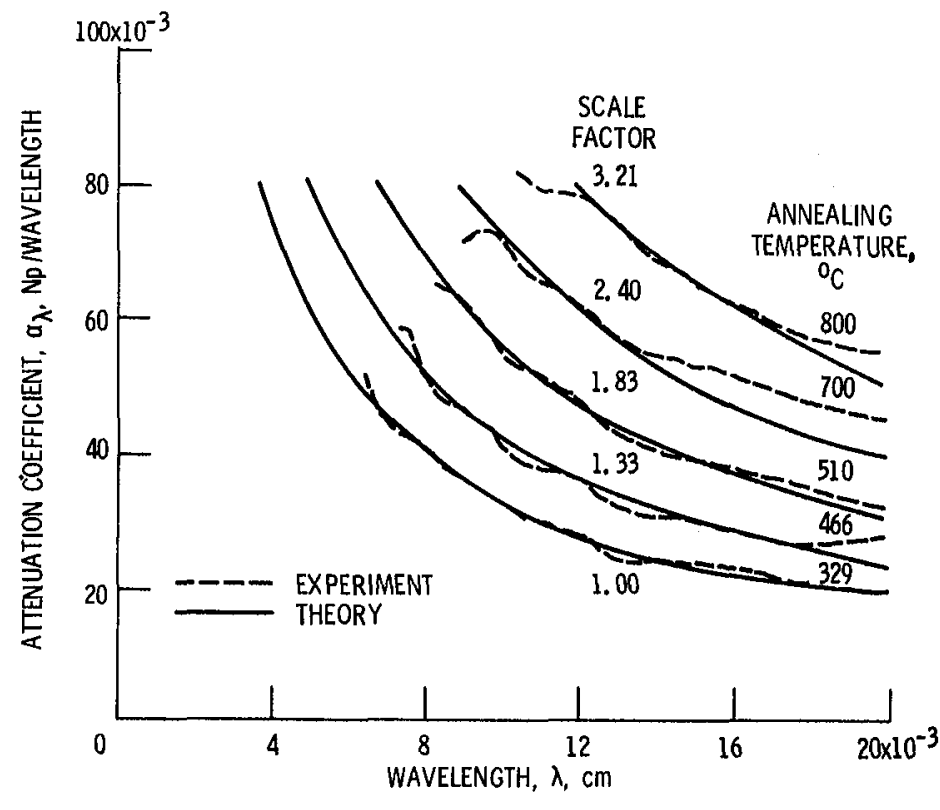

Figure 7. - Attenuation coefficient as function of wavelength for copper samples. Annealing time, $5 \mathrm{~min}$.

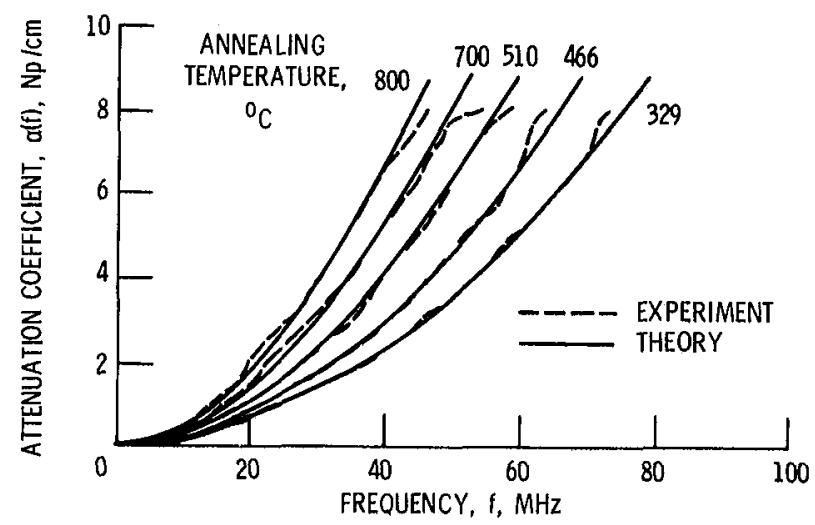

Figure 8. - Comparison between observed and theoretical attenuation coefficients as function of frequency for copper samples. Annealing time, $5 \mathrm{~min}$. 


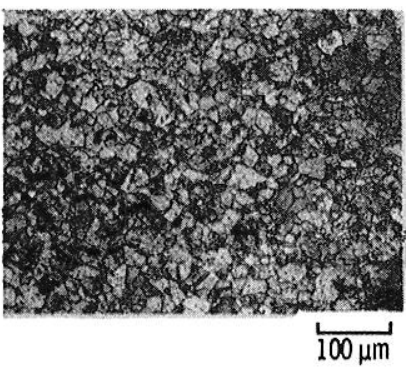

(a) Annealed for $2 \mathrm{~min}$ at $800^{\circ} \mathrm{C}$.

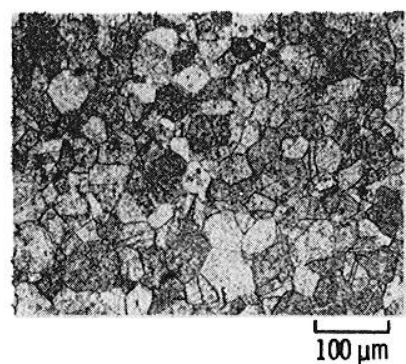

(b) Annealed for $5 \mathrm{~min}$ at $800^{\circ} \mathrm{C}$.
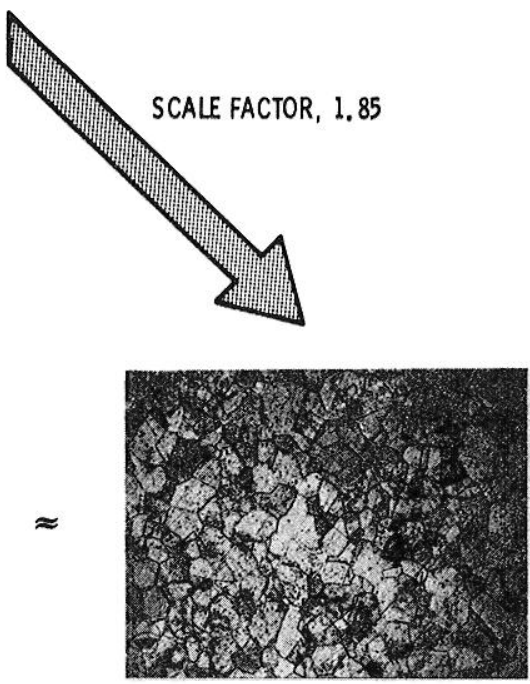

(c) 1.85 Magnification of figure $9(a)$.

Figure 9. - Photomicrographs of nickel sample.

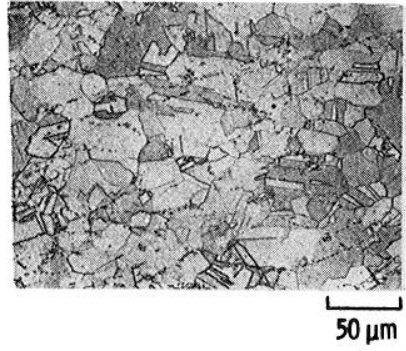

(a) Annealed for $5 \mathrm{~min}$ at $510^{\circ} \mathrm{C}$

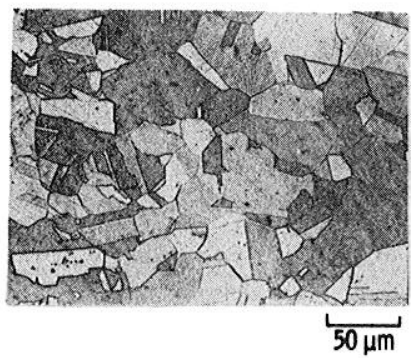

(b) Annealed for $5 \mathrm{~min}$ at $800^{\circ} \mathrm{C}$.
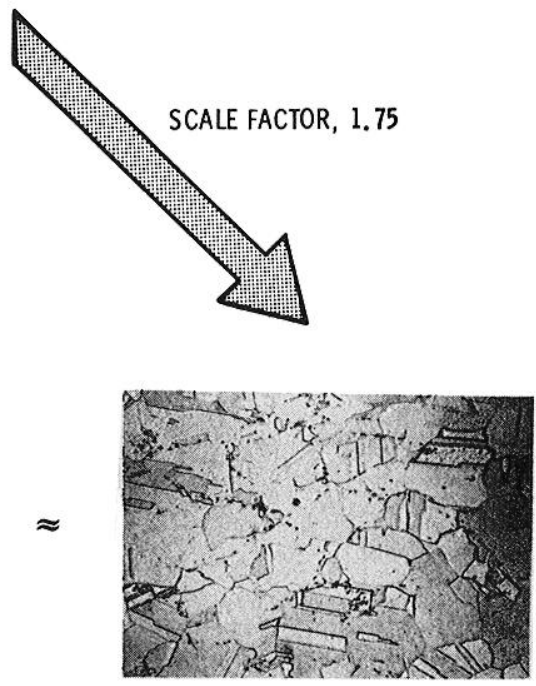

(c) 1.75 Magnification of figure 10(a).

Figure 10. - Photomicrographs of copper samples. 